\title{
Packable composites in UK general practices
}

\author{
Two-year evaluation of restorations of a packable composite placed in UK general dental practices \\ F. J. T. Burke, R J Crisp, M. Balkenhol, T. J. Bell, J. J. Lamb, K. McDermott, C. Siddons and B. Weller \\ Br Dent J 2005; 198: 293-296
}

\section{Objective}

The aim of this study was to assess the clinical performance at two years of 100 Solitaire 2 restorations placed in five United Kingdom dental practices by members of a practice-based research group.

\section{Method and materials}

Restorations were assessed after two years by a trained evaluator and the dental practitioner who had placed the material, for anatomic form, marginal adaptation, surface roughness, gingival condition and the presence or absence of secondary caries. In addition, the patients completed a questionnaire requesting details of the comfort and performance of the Solitaire 2 restoration(s).

\section{Results}

A total of 88 ( 58 Class II and 30 Class I) restorations of Solitaire 2 placed in 49 patients (mean age 43 years) were assessed. Twelve restorations could not be evaluated because of patient unavailability for the dates of the examinations. Two Class II restorations (2\%) had failed by the time of the two-year evaluation and the remaining 86 restorations were found to be intact with no secondary caries. A high percentage of optimal scores were recorded for anatomic form and surface roughness. The colour match of two restorations (2\%) was recorded as an obvious mismatch, but otherwise no unacceptable scores were recorded.

\section{Conclusions}

After two years of clinical service a high proportion (96\%) of the Solitaire 2 restorations that were available for re-examination, placed in general dental practice settings, were found to be performing satisfactorily.

\section{IN BRIEF}

- Details a two-year practice-based clinical evaluation of a packable resin composite material has been carried out.

- A good recall rate was obtained.

- The material was found to be performing satisfactorily in general practice patients.

\section{COMMENT}

This paper comments on the clinical evaluation of Solitaire 2, a packable composite. The restorations were placed and reviewed by members of the PREP panel (Product Research and Evaluation Panel). This is group of practitioners who, working together with university staff, evaluate new materials and techniques. Although the methods are not as rigorous as those demanded by a full scientific trial, the fact the materials are used in their normal setting, ie by dentists in dental practice, gives the findings a realistic validity. In this study over 100 restorations were placed and 89 examined at the two-year recall. Only two of the reviewed restorations had been replaced and there was no evidence that the restorations that had not been reviewed had failed. It appeared that some of the patients had been unable to attend the recall visit.

The results show that the performance of Solitaire 2 over a twoyear period was acceptable. In fact, this finding would be expected from any new composite because there is worldwide evidence that posterior composites can provide good clinical service for 10 years and beyond. For this reason two-year evaluations can only show whether a material is underperforming in relation to the expected behaviour for a genus of material. That is not to criticise this study because, while manufacturers are always trying to improve materials, there is always the possibility their modifications could have a detrimental effect which may only become apparent in a clinical evaluation. Clearly, that was not the case with Solitaire 2, but as stated above, the results do not show superiority over other materials.

The work of panels such as the PREP team will become increasingly important because of the relatively good performance of modern materials. To prove that any material is superior over its competitors it will be necessary to place a very large number (hundreds) of restorations and this will not be possible in a hospital environment. Also, as discussed in the article, evidencebased dentistry must be based on evidence from the use of the materials in practice rather than their use in the refined environment of a hospital trial.

Dr L.H. Mair

Senior Lecturer, Honorary Consultant, Head of Department, University of Liverpool doi: 10.1038/sj.bdj.4812656 